REVIEW

\title{
Neonatal pneumonia in developing countries
}

\section{T Duke}

Arch Dis Child Fetal Neonatal Ed 2005;90:F211-F219. doi: 10.1136/adc.2003.048108

Pneumonia contributes to between 750000 and 1.2 million neonatal deaths and an unknown number of stillbirths each year world wide. The aetiology depends on time of onset. Gram negative bacilli predominate in the first week of life, and Gram positive bacteria after that.

Streptococcus pneumoniae probably causes about $25 \%$ of neonatal pneumonia. Interventions that would reduce mortality from this condition would have a large range of beneficial effects: improved maternal health, better management of other common neonatal conditions, and reduced long term childhood and adult morbidity.

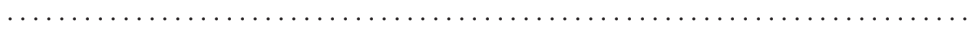

I is estimated that 3.9 million of the 10.8 million deaths in children annually world wide occur in the first 28 days of life. ${ }^{1}$ More than $96 \%$ of all neonatal deaths occur in developing countries, and pneumonia accounts for a substantial proportion of these. Intrauterine and early onset pneumonia was found at autopsy in $10-38 \%$ of stillborn and 20-63\% of liveborn babies who subsequently died (with all but one of six studies in the range of $20-32 \%){ }^{2}$ In a separate series of 1044 autopsies on neonatal deaths in the first 48 hours of life, $20-38 \%$ had pneumonia, with the highest incidence in poorer socioeconomic groups. ${ }^{3}$ There are therefore likely to be between 750000 and 1.2 million neonatal deaths annually where pneumonia is involved, ${ }^{4}$ and neonatal pneumonia accounts for $10 \%$ of global child mortality.

Throughout childhood, the greatest risk of death from pneumonia is in the neonatal period. In a field trial of community based management of childhood pneumonia in India, more than half of all child deaths from pneumonia occurred among neonates. ${ }^{5}$ Pneumonia mortality risk is strongly dependent on birth weight and age of onset. Case fatality rates are much higher for intrauterine or early onset pneumonia than for late onset neonatal pneumonia, ${ }^{67}$ and higher among low birthweight newborns. ${ }^{8}$

The proportion of neonatal respiratory distress that is caused by pneumonia will depend on where the source population is from (tertiary hospital, district hospital, or community), the stage in the perinatal period, the gestational age of the babies and the availability of intensive care, and the definition of pneumonia. Of 150 neonates with respiratory distress presenting to a referral hospital in India, 103 (68.7\%) were diagnosed to have pneumonia. ${ }^{9}$ Using a different case definition in a teaching hospital in Brazil, of 318 infants presenting with respiratory distress within the first 4 days of life, bacterial infection was proven on culture in $31(9.7 \%)$, and another $62(19.5 \%)$ had radiographic signs of pneumonia. $^{10}$

\section{CLASSIFICATION AND PROBLEMS IN UNDERSTANDING THE EPIDEMIOLOGY OF NEONATAL PNEUMONIA}

Neonatal pneumonia can be arbitrarily classified as early and late onset. There are various definitions of early onset pneumonia; some authors have used 48 hours as a cut off, others have suggested 7 days. Because of the way some aetiology studies have been reported, and the differences in aetiology, it may be operationally useful to separate the disease classification between the first week of life and the subsequent three weeks. In most series, Gram negative bacteria predominate in the first week and Gram positive bacteria predominate subsequently.

Intrauterine pneumonia can be considered a subgroup of early onset disease; this presents as stillbirth, low Apgar scores, or severe respiratory distress at the time of birth and is commonly associated with chorioamnionitis. Intrauterine and early onset pneumonia is acquired by transplacental spread or from aspiration of infected amniotic fluid after prolonged rupture of membranes or during delivery. Intrauterine pneumonia also occurs in the setting of a systemic infection in the mother, such as rubella, cytomegalovirus, Treponema pallidum, Listeria monocytogenes, tuberculosis, and HIV. These infections may be asymptomatic in the mother. Associated features include hepatosplenomegaly, thrombocytopenia, and jaundice.

Pneumonia acquired from bacterial infection of amniotic fluid or colonisation of the birth canal is often associated with chorioamnionitis and evidence of fetal asphyxia. How asphyxia relates to pneumonia is not certain, but it can cause fetal gasping and aspiration of contaminated amniotic fluid. That pneumonia occurs from aspiration is supported by the common finding of amniotic fluid debris or maternal leucocytes in the histological examination of the lungs.

It can be difficult to distinguish early onset pneumonia from hyaline membrane disease; the clinical picture and radiographic appearance can be identical, and in many settings radiology and bacteriology are unavailable. Gestational age and onset of symptoms provide clues in many cases. However, hyaline membrane disease also occurs close to term (in infants of diabetic mothers, and rarely surfactant protein deficiency), intrauterine acquisition of infection often results in premature onset of labour, and gestational age assessments are often not possible in settings where antenatal care is minimal and health workers are 
Table 1 Sensitivity (\%) of clinical signs for radiographically diagnosed pneumonia in neonates in developing countries

\begin{tabular}{lllllll}
\hline Study & $\begin{array}{l}\text { Chest } \\
\text { recession }\end{array}$ & Tachypnoea & Fever & $\begin{array}{l}\text { Inability } \\
\text { to feed }\end{array}$ & Cyanosis & Cough \\
\hline Singhi and Singhi ${ }^{15}$ & 84 & 89 & 56 & 49 & 12 & 84 \\
Misra et af ${ }^{4}$ & 91 & 72 & 30 & 43 & 39 & 30 \\
Shakunthala et al & 36 & 60 & - & 46 & 40 & 36 \\
\hline
\end{tabular}

not trained in this type of clinical scoring. Therefore basing treatment decisions for respiratory distress in the newborn on estimated gestational age is impractical and unsafe. The difficulty in distinguishing pneumonia from hyaline membrane disease and other non-infective respiratory conditions of the newborn-for example, transient tachypnoea of the newborn-presents a problem in understanding the contribution of pneumonia to mortality in the first week of life.

The literature on the bacterial aetiology of neonatal pneumonia is influenced by studies that include nosocomial infection in neonatal intensive care units. ${ }^{11}{ }^{12}$ Many studies do not report the age of onset. A further difficulty is that blood cultures will underestimate the proportion of pneumonia that is bacterial, and lung puncture studies have rarely been performed on neonates. ${ }^{36}$ In Ethiopia, a diagnosis of pneumonia in young infants was negatively associated with bacteraemia, compared with the diagnoses of clinical sepsis, "severe disease", or "eventually fatal sepsis". ${ }^{13}$ In studies where a high proportion of infants have previously received antibiotics, the highest yield has come from the combination of blood culture, lung puncture culture, and serum and lung aspirate latex agglutination antigens. ${ }^{14}$

The multi-country World Health Organisation young infant study has provided the most useful data yet on the aetiology of community acquired neonatal sepsis, including pneumonia from a range of developing countries. However, this study predominantly recruited young infants from outpatient departments in hospitals. This limited the number of neonates recruited from, or soon after, birth; thus intrauterine pneumonia and early onset pneumonia, which present in the first 48 hours of life, was not as well represented.

The following assumptions may be valid. The epidemiology of neonatal pneumonia and implications for treatment and prevention are sufficiently similar to those of neonatal bacteraemia, meningitis, and other forms of sepsis that blood culture and cerebrospinal fluid isolates can be used to understand the aetiology, notwithstanding the underestimation of pneumonia disease incidence without lung aspirates. Data on the aetiology of chorioamnionitis can be used to understand the aetiology of intrauterine and early onset pneumonia.

Table 2 Yield from cultures of lung puncture on 755 neonates who were stillborn or died in the first 72 hours of life ${ }^{3}$

\begin{tabular}{ll}
\hline Bacteria & Number \\
\hline Escherichia coli & 71 \\
Aerobacter aerogenes & 45 \\
Streptococcus beta haemolytic & 29 \\
Pseudomonas aeruginosa & 27 \\
Streptococcus viridans & 21 \\
Staphylococcus aureus & 17 \\
Proteus vulgaris & 11 \\
Streptococcus non haemolytic (Group D) & 8 \\
\hline
\end{tabular}

\section{CLINICAL FEATURES}

A definition of neonatal pneumonia was proposed in one Indian study (shown in the box), ${ }^{9}$ but none have been prospectively validated. This complex algorithm has limited application: at best in tertiary settings and as a standardised research tool. In most health facilities, laboratory testing, and often radiology, are unavailable.

In primary and many secondary healthcare facilities, it is sensible to consider neonatal pneumonia within the spectrum of neonatal sepsis. For practical purposes, WHO does not distinguish between neonatal pneumonia and other forms of severe sepsis, such as bacteraemia or urinary tract infection. There is much overlap in clinical signs and organ involvement, and empirical treatment regimens are similar.

Studies that have evaluated the clinical signs of radiographically proven neonatal pneumonia have reported some consistent and some apparently contradictory findings (table 1). Tachypnoea is present in $60-89 \%$ of cases. Chest recession is present in over $80 \%$ of cases in most studies, although one ${ }^{6}$ found that subcostal and intercostal retractions were only present in $36 \%$ of cases. Cough was absent in

Definition of neonatal pneumonia used in one Indian study 9

A neonate with respiratory distress (any of: rapid, noisy, or difficult breathing; respiratory rate $>60 / \mathrm{min}$; chest retractions; cough; grunting) who has: (a) a positive blood culture or (b) any two or more of the following:

(1) Predisposing factors

- Maternal fever $\left(>38^{\circ} \mathrm{C}\right)$

- Foul smelling liquor

- Prolonged rupture of membranes (>24 hours)

(2) Clinical picture of sepsis

- Poor feeding

- Lethargy

- Poor reflexes

- Hypothermia or hyperthermia

- Abdominal distension

(3) Radiograph suggestive of pneumonia (nodular or coarse patchy infiltrate, diffuse haziness or granularity, air bronchogram, lobar or segmental consolidation); radiological changes not resolved within 48 hours

(4) Positive sepsis screen (any of the following):

- Bands $>20 \%$ of leucocytes

- Leucocyte count out of reference range

- Raised C reactive protein

- Raised erythrocyte sedimentation rate 
about two thirds of cases in two studies, ${ }^{6}{ }^{14}$ but in less than $20 \%$ of cases from one study. ${ }^{6}$ For the purposes of designing primary care treatment strategies, Singhi and Singhi ${ }^{15}$ suggested that the standard definition for neonatal pneumonia could be "cough and chest wall recession" or "a history of rapid breathing and chest wall retraction". Use of cough and respiratory rate $>50$ breaths/min as the definition would have missed radiographically proven pneumonia in $25 \%$ of the cases in their series.

\section{FACTORS PREDICTING EARLY ONSET PNEUMONIA}

Predictive factors may be absent from many neonates with early onset pneumonia. A study of neonates in the United Kingdom with early onset pneumonia found that risk factors were present in $78 \%$, but in almost half ( 17 of 35 ) the only predictor was early onset of labour. ${ }^{16}$ In India, only 40\% (42 of 103) of mothers of neonates with pneumonia had identifiable risk factors: fever, prolonged rupture of membranes (>24 hours), leaking amniotic fluid, or foul smelling liquor. ${ }^{9}$ A problem with the methodology of the latter study is that known risk factors are used as one criterion to define pneumonia (box), therefore investigation of the proportion of neonatal pneumonias with these factors is a circular argument. Nevertheless, in this study from India, $>50 \%$ of neonates with an eventual diagnosis of pneumonia had no known predisposing factors. This suggests that other criteria used to diagnose pneumonia in this study were non-specific, and the proportion of neonatal respiratory distress that was ascribed to pneumonia $(68 \%)$ is an overestimate, or that some predisposing factors for neonatal pneumonia are difficult to recognise.

\section{AETIOLOGY AND PATHOPHYSIOLOGY Intrauterine pneumonia}

In an investigation of the aetiology of chorioamnionitis and perinatal infection, cultures of lung puncture and aspirate were carried out on 755 neonates who were stillborn or died in the first 72 hours of life. ${ }^{3}$ Table 2 lists the bacteria isolated.

\section{Early onset neonatal pneumonia}

Early onset neonatal pneumonia results from infection acquired by aspiration after early rupture of membranes or during labour, or an initially lower grade intrauterine infection associated with maternal chorioamnionitis (but
Table 4 Bacterial pathogens isolated from blood culture or cerebrospinal fluid in 1313 neonates aged 7-29 days in the multi-centre WHO young infant study, ${ }^{17}$ and in 260 neonates 8-30 days in Kenya

\begin{tabular}{llll}
\hline Bacterial pathogens & $\begin{array}{l}\text { WHO young } \\
\text { infant study }\end{array}$ & $\begin{array}{l}\text { English } \\
\text { et af }\end{array}$ & Total \\
\hline Total & 1313 & 260 & 1573 \\
Total positive cultures & $64(4.9 \%)$ & $22(8.5 \%)$ & $86(5.5 \%)$ \\
S pyogenes & 14 & 5 & 19 \\
Staph aureus & 15 & 3 & 18 \\
S pneumoniae & 8 & 4 & 12 \\
Escherichia coli & 10 & 1 & 11 \\
Salmonella spp & 8 & 0 & 8 \\
Group B Streptococcus & 2 & 6 & 8 \\
Klebsiella pneumoniae & 2 & 0 & 2 \\
Enterobacter & 2 & 0 & 2 \\
H influenzae & 1 & 1 & 2 \\
Group G Streptococcus & 1 & 0 & 1 \\
Acinetobacter & 1 & 0 & 1 \\
Pseudomonas & 0 & 1 & 1 \\
Enterococcus spp & 0 & 1 & 1 \\
\hline & & & \\
\hline
\end{tabular}

not of a magnitude sufficient to cause death or severe respiratory distress at birth). Table 3 lists the bacterial causes found in studies from developing countries that clearly distinguished early from late onset neonatal sepsis and pneumonia. Escherichia coli, group B Streptococcus, Klebsiella spp, Staphylococcus aureus and Streptococcus pneumoniae were the most commonly isolated bacteria. In a further study that did not make a clear distinction between early and late causes, among 150 Indian neonates presenting at a median of 164 hours of life with respiratory distress, of whom 103 were diagnosed with pneumonia, blood cultures were positive in 49 cases (48\%): Klebsiella in 28, Staph aureus in seven, coagulase negative Staphylococci in seven. ${ }^{9}$

\section{Late onset neonatal pneumonia}

Late onset neonatal pneumonia needs to be distinguished from pulmonary oedema secondary to a congenital heart lesion, and rarely from other pathology such as pulmonary haemorrhage or infarct. To understand the aetiology of late onset, community acquired neonatal pneumonia there is a need to exclude series from neonatal units in Western

Table 3 Cause of early neonatal sepsis and pneumonia in developing countries

\begin{tabular}{|c|c|c|c|c|}
\hline Bacteria & $\begin{array}{l}\text { WHO young } \\
\text { infant study }\end{array}$ & $\begin{array}{l}\text { English } \\
\text { ef }\left.a\right|^{18}\end{array}$ & $\begin{array}{l}\text { Mussi-Pinhata } \\
\text { ef } a f^{\circ}\end{array}$ & Total \\
\hline No of patients & 360 & 432 & 261 & 1053 \\
\hline Total positive cultures & $26(7.2 \%)$ & $40(9.3 \%)$ & $25(9.6 \%)$ & $91(8.6 \%)$ \\
\hline E coli & 6 & 8 & 4 & 18 \\
\hline Group B Streptococcus & 1 & 6 & $\begin{array}{l}6 \text { (+8 with positive GBS } \\
\text { urinary antigen) }\end{array}$ & $\begin{array}{l}13 \text { (+8 with positive } \\
\text { GBS urinary antigen) }\end{array}$ \\
\hline Klebsiella spp & 0 & 10 & 0 & 10 \\
\hline Staph aureus & 5 & 1 & 3 & 9 \\
\hline$S$ pneumoniae & 2 & 3 & 3 & 8 \\
\hline Pseudomonas spp & 2 & 5 & 0 & 7 \\
\hline Proteus mirabilis & 0 & 4 & 2 & 6 \\
\hline Enterobacter & 4 & 0 & $\overline{0}$ & 4 \\
\hline Staph epidermidis & 0 & 0 & 4 & 4 \\
\hline Streptomyces pyogenes & 3 & 0 & 0 & 3 \\
\hline Enterococcus spp & 0 & 3 & 0 & 3 \\
\hline Haemophilus influenzae & 0 & 0 & 2 & 2 \\
\hline Group G Streptococcus & 1 & 0 & 0 & $\overline{1}$ \\
\hline Salmonella spp & 1 & 0 & 0 & 1 \\
\hline Acinetobacter & 1 & 0 & 0 & 1 \\
\hline Neisseria meningitidis & 0 & 0 & 1 & 1 \\
\hline
\end{tabular}

Bacterial pathogens isolated from blood culture or cerebrospinal fluid in the first week of life in 360 neonates from the multicentre WHO young infant study, ${ }^{17} 432$ neonates in the first week of life in Kenya, ${ }^{18}$ and in 261 neonates with respiratory distress in the first 4 days of life in Brazil.

GBS, Group B Streptococcus. 
countries, where colonisation of endotracheal tubes and breaches in local immunity lead to bacteria entering the lower respiratory tract. Until the WHO young infant study, ${ }^{8} 131719$ the aetiology of community acquired, late onset neonatal infection in developing countries had not been widely studied. Table 4 lists the major pathogens isolated from blood cultures or cerebrospinal fluid in neonates aged 7-29 days in this study and in a study from Kenya. Bacterial isolation in the WHO study was from blood cultures, cerebrospinal fluid, pus swabs, and conjunctival swabs. Given that more than half of all childhood bacterial pneumonias are not associated with bacteraemia, this series of studies is likely to have underestimated the proportion of neonatal pneumonias that are bacterial in origin.

Two lung aspirate studies from India (table 5) have highlighted the importance of Gram positive bacteria in neonatal pneumonia: one study found $S$ pneumoniae (14 out of 50) and Staph aureus ( 14 out of 50) to be the most common causes of neonatal pneumonia. ${ }^{6}$ A second study found $S$ pneumoniae antigen positive in serum or lung aspirate of 10 of $44(22 \%)$ neonates with pneumonia (but no cultures of $S$ pneumoniae were positive), and Gram negative bacilli were cultured in 11 of $44(25 \%) .{ }^{14}$ The age of onset of infection in these two studies was not reported, but in a study of neonates with pneumococcal sepsis in the United States, the median age of onset was 18.9 days (SD 4.6). For those in whom pneumonia was the major site of infection, the age of onset was 12.3 days (SD 12.4). ${ }^{20}$ The yield from these two lung aspirate studies, one of which also used non-culture techniques, was $62 \%$ compared with less than $10 \%$ in other studies that relied on blood and cerebrospinal fluid bacterial cultures alone.

\section{Atypical pathogens}

Congenital syphilis is still a major cause of neonatal infection in many developing countries, and fatal cases are invariably associated with severe pneumonitis and hypoxaemia (pneumonia alba). In a study from Papua New Guinea, ${ }^{21}$ congenital syphilis was the cause of death in $13.5 \%$ ( 17 of 126) neonates, and the case fatality rate for congenital syphilis was 33\%. ${ }^{22}$ Severe hypoxaemia was common. ${ }^{23}$ In Malawi, independent risk factors for neonatal death included maternal syphilis (hazard ratio $2.4 ; 95 \%$ confidence intervals 1.3 to 4.4 ), low birth weight, and first pregnancy. ${ }^{24}$

Chlamydia trachomatis may contribute substantially to late onset neonatal pneumonia in countries where untreated sexually transmitted infections are common. The evidence for this is circumstantial, but suggestive of a major effect. In Papua New Guinea, evidence of $C$ trachomatis was found by direct immunofluorescence in $22 \%$ ( 138 of 638) of nasopharyngeal aspirates from young infants presenting to an

Table 5 Lung aspirate studies in neonatal pneumonia

\begin{tabular}{llll}
\hline Bacteria & $\begin{array}{l}\text { Shakunthala } \\
\text { et } \mathbf{a l}^{\mathbf{6}}\end{array}$ & $\begin{array}{l}\text { Misra } \\
\text { ef } \mathbf{a} \mathbf{f}^{\mathbf{4}}\end{array}$ & Total \\
\hline Total & 50 & 44 & 94 \\
Positive isolate & $31(62 \%)$ & $27(61 \%)$ & $58(62 \%)$ \\
S pneumoniae & 14 & $10^{*}$ & 24 \\
Staph aureus & 14 & 0 & 14 \\
Klebsiella & 0 & 5 & 5 \\
Sepidermidis & 0 & 4 & 4 \\
Streptococcus & 2 & 2 & 4 \\
Pseudomonas & 1 & 2 & 3 \\
E coli & 0 & 1 & 1 \\
Salmonella & 0 & 1 & 1 \\
Morganella & 0 & 1 & 1 \\
Enterobacter & 0 & 1 & 1
\end{tabular}

*S pneumoniae isolated from lung puncture using counterimmunoelectrophoresis antigen testing. outpatient's department. Positive $C$ trachomatis nasopharyngeal aspirates were found in 33\% (100 of 303) of infants with severe pneumonia, $18 \%$ (38 of 217 ) of those with non-severe pneumonia, and 9\% (26 of 295) of young infants with no pneumonia, sepsis, meningitis, or conjunctivitis. The presence of Chlamydia was associated with radiographic evidence of pneumonia as well as hypoxaemia, and there was a borderline association of positive $C$ trachomatis nasopharyngeal aspirates and pneumococcal bacteraemia. ${ }^{25}$ Recovery rates were high $(>85 \%)$ despite antibiotics against Chlamydia being rarely used, suggesting either that Chlamydia predisposes, perhaps by mucosal injury, to secondary bacterial infection, which is more serious in the setting of co-infection, but still responds adequately to conventional antibiotics or Chlamydia responds sufficiently to commonly used antibiotics for young infant sepsis.

Among Kenyan neonates aged 7-30 days with pneumonia, 24 of $52(46 \%)$ had the combination of both interstitial pneumonia and colonisation of the upper airways with $C$ trachomatis. ${ }^{26}$ In Ethiopia, of 202 infants $<3$ months of age presenting to an outpatient's department, 32 (15.8\%) had a nasopharyngeal aspirate positive for $C$ trachomatis. ${ }^{13}$

On the basis of pooled results from 10 studies, the estimated risk of Chlamydia pneumonia developing in an infant whose mother is colonised with $C$ trachomatis is $7 \%$ (95\% confidence interval $5.1 \%$ to $9.4 \%$ ). ${ }^{27}$ The two largest cohort studies of infants exposed to $C$ trachomatis reported pneumonia incidences of $3 \%{ }^{28}$ and $16 \% .{ }^{29}$ No studies have used the optimum method for determining the cause of pneumonia: isolation of $C$ trachomatis from a percutaneous lung aspirate.

Apart from intrauterine infections, the viral aetiology of neonatal pneumonia has not been systematically studied. Respiratory syncitial virus has been shown to be a common pathogen in infants under 6 months of age, ${ }^{3031}$ and it is likely that this virus is an important cause of seasonal late onset neonatal pneumonia in some settings in developing countries.

\section{HIV and neonatal pneumonia}

There are several reasons why HIV infection is a risk factor for the development of neonatal pneumonia and increases the risk of mortality from the latter. Although HIV infection usually becomes manifest clinically in later infancy, a rapidly progressive congenital form of HIV associated with persistent pneumonia in the neonatal period has been described from South Africa and Zimbabwe. ${ }^{32} 33$ This is associated with hepatosplenomegaly, lymphadenopathy, prematurity, and growth retardation. Co-infection with tuberculosis, syphilis, and cytomegalovirus are common and may be responsible for the early manifestations of this disease. ${ }^{32}{ }^{34}$ Maternal HIV infection increases the probability of death from neonatal respiratory distress syndrome and predisposes to sepsis from streptococci and staphylococci. ${ }^{33}$ Among mothers and infants in Pune, India using cow, goat, or buffalo milk as a substitute for human milk to reduce the risk of mother to child HIV transmission, the rate of hospital admission in the neonatal period from pneumonia (and other infections) was much higher than for the neonates of HIV positive mothers who breast fed. ${ }^{35}$

\section{ROLE OF HYPOXAEMIA IN NEONATAL PNEUMONIA}

Hypoxaemia is a common complication of neonatal illness, because of primary respiratory disease (pneumonia, hyaline membrane disease, transient tachypnoea of the newborn) and because apnoea is a common non-specific response to sepsis, hypothermia, and hypoglycaemia. There have been few investigations of the incidence of hypoxaemia in neonatal illness in developing countries. The incidence varies 
Table 6 Reported case fatality rates for neonatal pneumonia

\begin{tabular}{|c|c|c|c|c|c|}
\hline Study & $\begin{array}{l}\text { Country and type of } \\
\text { presentation }\end{array}$ & Number & Deaths & $\begin{array}{l}\text { Number of cases } \\
\text { in 1st week/deaths }\end{array}$ & Risk factors \\
\hline Misra et al ${ }^{14}$ & India; university hospital & 44 & $14(32 \%)$ & NR & Slow respiratory rate; low birth weight \\
\hline Shakunthala ef al ${ }^{6}$ & $\begin{array}{l}\text { India; university hospital } \\
\text { neonatal unit }\end{array}$ & 50 & $24(48 \%)$ & $23 / 17$ & First week presentation; low birth weight \\
\hline Mathur et al ${ }^{9}$ & $\begin{array}{l}\text { India; university hospital } \\
\text { neonatal unit }\end{array}$ & 103 & $32(31 \%)$ & NR & $\mathrm{A}-\mathrm{aDO}_{2}>250 \mathrm{~mm} \mathrm{Hg}$ \\
\hline Bang et al ${ }^{45}$ & $\begin{array}{l}\text { India; community based } \\
\text { intervention study }\end{array}$ & 65 & $10(15 \%)$ & NR & $\begin{array}{l}\text { Referral indications: stridor, unconsciousness, } \\
\text { convulsions or inability to suck }\end{array}$ \\
\hline Singhi \& Singhi ${ }^{15}$ & $\begin{array}{l}\text { India; university hospital } \\
\text { emergency department } \\
\text { presentations }\end{array}$ & 89 & $7(8 \%)$ & NR & Slow respiratory rate \\
\hline Webber et al ${ }^{7}$ & $\begin{array}{l}\text { UK; university hospital } \\
\text { neonatal unit }\end{array}$ & 76 & $11(14 \%)$ & $35 / 10$ & First week presentation \\
\hline Total & & 427 & $98(23 \%)$ & & \\
\hline
\end{tabular}

with the level of health facility. Hypoxaemia was present in $38.5 \%$ of infants under 2 months of age presenting to a tertiary hospital emergency department in India $\left(\mathrm{SpO}_{2}<90\right)^{36}$ and in $43 \%$ of neonates admitted to a provincial hospital in Papua New Guinea $\left(\mathrm{SpO}_{2}<86 \%\right.$, more than 2SD below the population mean at $1600 \mathrm{~m}) .^{23}$ In Papua New Guinea, hypoxaemia was present in $77 \%$ of neonates with pneumonia, but also in $36 \%$ of neonates without clinically definable acute lower respiratory tract infection..$^{23}$ Because of referral of more seriously ill infants, a higher proportion of neonates seen at tertiary hospitals than at primary care facilities will have hypoxaemia. However, hypoxaemia is a common feature of neonatal illness even in primary care facilities. Among infants less than 2 months of age presenting to eight remote rural primary health centres in Papua New Guinea, one or more signs of severe respiratory compromise were present in $25 \%$ (128 of 511): fast respiratory rate (102); severe chest indrawing (74); cyanosis (35); or apnoea (32). Each of these signs was associated with an increased risk of death. Pulse oximetry was not performed in this study; however, 49 infants $(9.6 \%)$ had cyanosis or apnoea, two of the most specific clinical signs for neonatal hypoxaemia. ${ }^{23}$ Detection of hypoxaemia in neonates using clinical signs is difficult. In a study in Papua New Guinea, cyanosis was the most sensitive sign, but will still miss up to one quarter of neonates with hypoxaemia. A respiratory rate within the normal range of 30-60 breaths/min was found in just over half of all neonates with hypoxaemia. Incidences of hypoxaemia of $10 \%$ in primary care facilities and more than $30 \%$ at hospitals of first referral represent huge numbers globally, and need for oxygen is rarely matched by available resources.

\section{OUTCOME}

Table 6 summarises published case fatality rates in community based and hospital based studies, most of which come from India. Mortality in early neonatal pneumonia is much higher $(74 \%$ and $29 \%$ in the two series that reported case fatality rate by age) than for late onset disease. Risk factors for death from neonatal pneumonia are early onset disease, hypoxaemia, low birth weight, ${ }^{6814}$ and absence of tachypnoea (table 7).

\section{TREATMENT}

There are no published randomised treatment trials of neonatal pneumonia, and few for neonatal sepsis in developing countries. ${ }^{37}$ Empirical treatment decisions must therefore be based on local resistance patterns and the costs, benefits, and availability of various antibiotic regimens. There are also some informative trials using retrospective or contemporaneous non-randomised control groups in communities and hospitals in developing countries.

\section{Antibiotics}

For the management of neonatal sepsis, WHO recommends giving ampicillin $(50 \mathrm{mg} / \mathrm{kg})$ every 12 hours in the first week of life and every eight hours from 2-4 weeks, plus a single daily dose of gentamicin. ${ }^{39}$ First line alternatives to ampicillin are benzylpenicillin or amoxicillin, and alternatives to gentamicin are kanamycin or streptomycin. Where there is strong evidence of staphylococcal infection (skin pustules, cellulitis, umbilical cord infection, pneumatocoeles, or empyema), cloxacillin or flucloxacillin, where these are available, should be substituted for ampicillin.

A daily dose regimen for gentamicin was piloted in a randomised trial in Kenyan infants less than 3 months of age. A regimen that consisted of a loading dose of $8 \mathrm{mg} / \mathrm{kg}$ followed by $2 \mathrm{mg} / \mathrm{kg}$ (if weight $<2 \mathrm{~kg}$ ) or $4 \mathrm{mg} / \mathrm{kg}$ (if weight $>2 \mathrm{~kg}$ ) in the first week of life, or $4 \mathrm{mg} / \mathrm{kg}$ (weight $<2 \mathrm{~kg}$ ) or $6 \mathrm{mg} / \mathrm{kg}$ (weight $>2 \mathrm{~kg}$ ) resulted in a higher proportion of initial gentamicin concentrations within the therapeutic range and a lower rate of potentially toxic gentamicin concentrations, than a three times a day dose schedule. ${ }^{37}$

Pus, from empyema or skin pustules, should be examined after Gram staining, where laboratory facilities are available. The yield from blood cultures is low, and culture facilities will not be available in most health centres and secondary referral hospitals where neonates present with pneumonia, but where possible blood for culture should be taken before

Table 7 Data showing that absence of tachypnoea is a risk factor for mortality

\begin{tabular}{llll}
\hline \multirow{2}{*}{ Study } & \multicolumn{4}{l}{ Number in RR range who died/total number in RR range (CFR) } \\
\cline { 2 - 4 } & $>\mathbf{6 0}$ breaths/min & $\mathbf{5 0 - 6 0}$ breaths/min & $<\mathbf{5 0}$ breaths/min \\
\hline Mathur ef af & $27 / 91(30 \%)$ & $1 / 5(20 \%)$ & $4 / 7(57 \%)$ \\
Singhi \& Singhi ${ }^{15}$ & $4 / 78(5 \%)$ & - & $3 / 11(27 \%)$ \\
Misra et al ${ }^{4}$ & $7 / 12(21 \%)$ & $7 / 32(58 \%)$ & - \\
\hline RR, Respiratory rate; CFR, RR specific case fatality rate. & \\
\hline
\end{tabular}


antibiotics are given, to guide second line antibiotic treatment in the event of treatment failure.

For neonates who are failing to respond to first line antibiotics, WHO suggests changing to chloramphenicol or a third generation cephalosporin. ${ }^{39}$ Chloramphenicol should not be used in premature infants, and should be avoided in the first week of life.

Antibiotic resistance among neonatal pathogens is increasing world wide. Reported rates of resistance depend partly on the proportion of cases that are acquired in hospitals, where multiresistant Pseudomonas, Klebsiella, Serratia, and other enteric Gram negative bacilli are more common than in community acquired sepsis. ${ }^{11}{ }^{40}$ Gentamicin resistance among Gram negative bacteria causing neonatal sepsis range from; $20 \%$ in Kenya; ${ }^{41} 24 \%$ in India; ${ }^{14} 43 \%$ in one study from Pakistan $^{42}$ and $78-84 \%$ in another; ${ }^{43} 66 \%$ in Papua New Guinea; ${ }^{40}$ and $77 \%$ in Guatemala. ${ }^{11}$ Despite high rates of reported resistance, the combination of ampicillin and gentamicin is still probably optimal first line treatment for severe community acquired neonatal pneumonia in most settings.

\section{Community based recognition and treatment}

Training village health workers to recognise the clinical signs of neonatal pneumonia has been shown to reduce total and pneumonia mortality in several projects in remote areas. A meta-analysis of five studies of community based trials from India, Pakistan, Bangladesh, Tanzania, and Nepal showed a pooled reduction in total mortality of $27 \%$ (95\% confidence interval $18 \%$ to $35 \%$ ) and a reduction in pneumonia mortality of $42 \%$ (95\% confidence interval $57 \%$ to $22 \%$ ). ${ }^{44}$

Antibiotics used in these studies included oral cotrimoxazole (for five days), ${ }^{55}$ procaine penicillin, and oral ampicillin. Investigators in India who originally used cotrimoxazole with substantial reductions in neonatal mortality, ${ }^{55}$ later used cotrimoxazole with a daily dose of gentamicin with apparently additional benefit. ${ }^{46}$

In these non-randomised community trials, supervision and community motivation was very high. There is a need to know if this approach can be translated to other developing countries.

\section{Oxygen and supportive care}

Major improvements to neonatal outcomes can occur by standardising provision of neonatal care in rural and district hospitals. In the Eastern Highlands of Papua New Guinea, where general improvements were made to neonatal care (better provision of oxygen, detection and treatment of apnoea, thermoregulation, detection and treatment of hypoglycaemia, protocols for the safe use of intravenous fluids, and improved nutrition), case fatality from pneumonia was more than 50\% lower during a 30 month period after the interventions compared with the 30 months before. ${ }^{47}$ Mortality also fell from other causes, particularly low birth weight, emphasising the values of a syndromic approach and general supportive measures rather than a diagnosis specific approach to neonatal care. These improvements occurred while WHO standard antibiotic treatment regimens were used throughout the two periods of study, and apart from oxygen and pulse oximetry did not require any intensive technologies.

Using oxygen, on the basis of objective evidence of hypoxaemia, has the potential for large reductions in neonatal and child mortality. ${ }^{48}$ Various methods are available for delivering oxygen, particularly cylinders of compressed gas and oxygen concentrators. Oxygen cylinders are costly and heavy to transport, but can be used anywhere. Oxygen concentrators are more cost effective in places remote from oxygen supply systems, but require a continuous power supply. A system of using concentrators with a cylinder as a back up in case of power failure may be optimal in rural hospitals in developing countries.

Appropriate attachments for delivering oxygen have recently been reviewed, ${ }^{49}$ in resource limited settings, use of nasal oxygen prongs is optimal but often limited by cost and availability. They do not require humidification, and low flow rates (0.5-2 litres/min) are effective in correcting hypoxaemia in most infants. A nasopharyngeal catheter is the most efficient method for delivering oxygen, providing the highest partial pressure of alveolar oxygen for a given flow rate; however, these are associated with higher complication rates than nasal prongs and do require humidification. A nasal catheter, inserted half way into the nasal passage, may be the most widely used method in developing countries: low flow rates are adequate, complications are rare, cost is a fraction of that of nasal prongs, and, where oxygen catheters are unavailable, nasogastric tubes are often used. Giving oxygen through a tube directed into a head box is inefficient in health facilities where oxygen supplies are limited. High flow rates ( $>4$ litres/min) are required to achieve a high fraction of inspired oxygen and to avoid carbon dioxide accumulation within a standard neonatal head box.

Many neonates with severe pneumonia will be unable to feed well in the acute stage of the illness and require nasogastric or intravenous fluids. Although there are no randomised trials to inform fluid management, in infants with bronchiolitis, free water excretion is noticeably reduced in the acute stage compared with during convalescence, suggesting activation of water conservation mechanisms. In animal models of pneumococcal sepsis, free water excretion is reduced to $25 \%$ of baseline values within hours of infection. Hyponatraemia is found in about $30 \%$ of cases of pneumonia in some series, is associated with high antidiuretic hormone secretion, and is more common in children with cyanosis, ${ }^{50}$ suggesting that the more severe the pneumonia, the better the physiological water conservation, and the less exogenous fluid required to maintain homoeostasis. Giving fluid by a nasogastric tube allows the continuation of breast milk feeding and maintenance of some energy intake, avoidance of hypoglycaemia and the complications of intravenous fluid administration, and is cheaper than intravenous fluids. This is preferred unless there is a true contraindication to feeding, such as frequent vomiting, intolerance of enteral feeds, or high risk of aspiration. In these circumstances, isotonic saline plus up to $10 \%$ dextrose run at lower than traditional maintenance rates may be most appropriate, and feeds introduced as soon as the contraindication to enteral feeding has resolved. ${ }^{51}$

\section{PREVENTION}

There are several reasons why interventions that can only be delivered in hospitals will make little impact on deaths from neonatal pneumonia. In many societies, neonates are not taken outside the home, even if they are sick, so referral to a hospital is impossible. Often there is a preference for traditional village remedies. In addition, because the signs of severe illness can be very subtle in neonates, parents may not recognise that their infant is sick until it is too late, and geographical and financial constraints make referral to hospital very difficult for much of the world's population. On the other hand, interventions solely at community level will not fully address the often fatal complications of hypoxaemia and apnoea. Improving supportive care and the safety and accessibility of hospitals for seriously ill newborn babies is a high priority globally. A comprehensive approach to prevention and treatment of neonatal pneumonia would therefore involve interventions at community level, in primary health facilities, and in district and tertiary hospitals. 
Some preventive interventions have been shown to lead to improved outcomes. Other interventions have shown efficacy in research or atypical circumstances, and there is a need to evaluate the feasibility in other settings. For some preventive interventions and treatment, there are sound reasons for further investigation of their potential role.

\section{PREVENTIVE MEASURES SHOWN TO BE EFFECTIVE} Active management of preterm rupture of membranes Antibiotic administration after preterm rupture of membranes is associated with a delay in delivery and a reduction in major markers of neonatal morbidity (neonatal infection, use of oxygen therapy, and abnormal cerebral ultrasound scans). ${ }^{52}$ Encouraging treatment and referral of mothers with preterm rupture of membranes would reduce the number of babies born with intrauterine and early onset pneumonia. ${ }^{53}$

\section{Promotion of basic newborn care in communities}

Early and exclusive breast feeding has been shown to decrease the risk of pneumonia in infants outside the neonatal period, and there is every reason to expect a similar or greater protective effect against late onset neonatal pneumonia. ${ }^{5455}$

\section{Prevention of nosocomial pneumonia}

Nosocomial pneumonia accounts for a large proportion of deaths from late onset pneumonia in industrialised countries and in developing countries with neonatal intensive care units. In these environments, infections due to enteric Gram negative bacilli (particularly Klebsiella, Enterobacter, and Pseudomonas), coagulase negative staphylococci, and, in some units methicillin resistant Staph aureus are common. ${ }^{56-58}$ Infections occur because of colonisation of endotracheal tubes, humidifier apparatus, ventilator tubing, and intravenous lines, and is transmitted by hands of clinical staff and on other equipment (stethoscopes, etc). The single most important intervention for infection control for protecting newborns from nosocomial sepsis is hand washing. ${ }^{59}{ }^{60}$ In addition, randomised trial evidence suggests that restricted antibiotic policies prevent nosocomial infections in neonates. In a multicentre randomised control trial, penicillin and tobramycin for early onset sepsis, and flucloxacillin and tobramycin for late onset sepsis resulted in much lower incidence of colonisation with resistant bacteria than the combination of ceftriaxone and amoxycillin. ${ }^{61}$ A regimen avoiding the use of amoxycillin and third generation cephalosporins restricts the resistance problem. Prevention of infection also requires the identification and cleaning of contaminated equipment.

\section{APPROACHES WARRANTING FURTHER INVESTIGATION \\ Maternal immunisation}

Giving pneumococcal polysaccharide vaccine to mothers provided protective antibodies in newborns against $S$ pneumoniae in the Philippines, Papua New Guinea, Bangladesh, and the Gambia. ${ }^{62-65}$ Antibodies are acquired through both the maternal-fetal circulation and breast milk. ${ }^{64}$ In the Gambia, pneumococcal antibodies were found to disappear rapidly during the first months of life; so, to achieve sustained protection, maternal vaccination would need to be supplemented by infant vaccination, and breast feeding promoted. In Papua New Guinea, there was evidence that vaccine response was not suppressed in older infants whose mothers had been vaccinated during pregnancy. Given the finding from limited lung aspirate studies that $25 \%$ of neonatal pneumonia is due to Pneumococcus, even protection in the first few months of life may be extremely beneficial, and the full potential of maternal immunisation should be fully explored. ${ }^{66}$ The possibilities increase for maternal immunisation, as more potential vaccine candidates become available, including herpes simplex, cytomegalovirus, and HIV vaccines. ${ }^{67}$

\section{Prevention and treatment of sexually transmitted infections}

Programmes to prevent and treat syphilis, Chlamydia, and HIV would substantially improve neonatal outcomes and reduce much of the neonatal respiratory disease caused by these infections. In a Kenyan maternity hospital, an antenatal treatment programme for syphilis based on rapid plasma reagin testing significantly improved pregnancy outcomes. ${ }^{68}$ However, tackling this issue is logistically difficult in countries where antenatal care is not accessed by most women, and where laboratory facilities in remote health clinics are absent or limited. Accurate rapid testing would have more utility than laboratory based serological tests, but rapid test kits are often expensive, of variable sensitivity, and field trials in developing countries are few. In South Africa and rural Gambia, the rapid plasma reagin test had positive predictive value (for subsequent confirmation of syphilis by laboratory based serological testing) of around 50\%, and sensitivity around $75 \% .{ }^{69}{ }^{70}$ Other rapid tests may have greater predictive power, but are more costly. Retesting in pregnancy may be required in some settings. ${ }^{71}$ There is an urgent need for further evaluation of rapid screening tests for use in health clinics.

\section{Cleansing the birth canal with antiseptic solution}

In a non-randomised controlled trial in Malawi, cleansing the birth canal with $0.25 \%$ chlorhexidine at every vaginal examination before delivery was associated with a lower rate of admissions for neonatal sepsis, lower mortality from infectious causes and lower overall neonatal mortality, and lower maternal postpartum infection rate, than when no cleansing was done. ${ }^{72}$

\section{Vitamin A supplementation at birth}

Two large randomised trials have shown that vitamin A supplementation at birth reduces young infant mortality. ${ }^{73}{ }^{74}$ Giving $24000 \mathrm{IU}$ vitamin A on day 1 and day 2 of life in India resulted in a $22 \%$ reduction in total mortality at 6 months of age; the greatest impact was in the period 2 weeks to 3 months of age, and in those of birth weight $<2000 \mathrm{~g}$. A possible reason for this is reduced $S$ pneumoniae colonisation in early infancy. Within this large randomised control trial, nasopharyngeal colonisation with Pneumococcus was less common at 4 months of age. ${ }^{75}$ In another trial in Indonesian newborn, the risk of death was 64\% lower (95\% confidence interval $13 \%$ to $84 \%$ ), and the incidence of cough and fever were significantly lower in the first 4 months in those who were given 50000 IU vitamin A on the first day of life. This may shift the age of first infection to later in infancy when mortality will be less, therefore there are major implications for the timing of the first dose of vitamin $\mathrm{A}$ in supplemental programmes.

\section{Management of apnoea and respiratory failure}

Apnoea is a common complication of neonatal pneumonia, occurring in $28-43 \%$ of cases, ${ }^{676}$ and respiratory failure unresponsive to oxygen invariably precedes a fatal outcome. In some hospitals, provision of mechanical ventilation may do more harm than good because staff are not experienced with its use, or because the combination of poor infection control practices and colonised endotracheal tubes means that nosocomial pneumonia will cause the deaths of more babies than mechanical ventilation would save. Underwater bubble continuous positive airway pressure $(\mathrm{CPAP})^{77}$ may have a role in the management of apnoea or severe 
respiratory failure in these settings. One non-randomised study, used either nasopharyngeal CPAP or head box oxygen in neonates less than $1200 \mathrm{~g}$ and less than 28 weeks gestation who were "refused admission" to a neonatal intensive care unit, and found a higher rate of survival to hospital discharge in those who received CPAP. ${ }^{76}$ This study was very small ( 21 babies in total), and few conclusions can be drawn either about the benefits ( survival of $45 \% v 20 \%$ in the control group) or the potential harms (intraventricular haemorrhage in $45 \%$ of the treatment group compared with 0 in the controls) of CPAP. Relatively inexpensive methods of delivering underwater bubble CPAP are now available and warrant trials in moderate sized hospitals in developing countries. CPAP may reduce the need for oxygen in hospitals where monitoring of oxygen toxicity is not feasible.

There is also evidence that in low birthweight neonates with apnoea, respiratory stimulants such as aminophylline or caffeine may be more effective than CPAP in preventing the need for mechanical ventilation, ${ }^{78}$ more effective than kinaesthetic stimulation in prevention of apnoea, ${ }^{79}$ and that caffeine is associated with fewer adverse events than aminophylline and is equally effective in the prevention of apnoea. ${ }^{80}$ Most studies have been conducted in preterm babies whose predominant pathology is hyaline membrane disease or apnoea of prematurity. Whether these drugs are beneficial for pneumonia in preterm and term babies has not been established in controlled trials.

\section{CONCLUSIONS}

The global burden of neonatal pneumonia is huge. Efficient interventions must be targeted at all levels of the health services and communities. Unlike for pneumonia in older infants and children, where effective interventions against two bacteria (S pneumonia and $H$ influenzae) will substantially reduce disease prevalence, the aetiology of neonatal pneumonia is more complex. Interventions that will reduce mortality from neonatal pneumonia will be broad based and have a range of general positive effects: improved maternal health, better management of other common neonatal conditions, and reduced long term childhood and adult morbidity. Rising rates of resistance to affordable antibiotics may mean that case fatality rates from neonatal pneumonia will increase in developing countries, emphasising the need to explore widely applicable preventive and treatment measures.

Competing interests: none declared

\section{REFERENCES}

1 Black RE, Moris SS, Bryce J. Where and why are 10 million children dying every year? Lancet 2003;361:2226-34.

2 Barnett ED, Klein JO. Bacterial infections of the respiratory tract. In: Remington JS, Klein JO, eds. Infectious diseases of the fetus and newborn infant. Pennsylvania: WB Saunders Co, 2001:1006-18.

3 Naeye RL, Dellinger WS, Blanc WA. Fetal and maternal features of antenatal bacterial infections. J Pediatr 1971;79:733-9.

4 The Child Health Research Project. Reducing perinatal and neonatal mortality: report of a meeting Baltimore, Maryland. Baltimore, 1999;3:6-12.

5 Bang AT, Bang RA, Tale O, et al. Reduction in pneumonia mortality and total childhood mortality by means of community-based intervention trial in Gadchiroli, India. Lancet 1993;336:201-6.

6 Shakunthala SKV, Rao GM, Urmila S. Diagnostic lung puncture aspiration in acute pneumonia of newborn. Indian Pediatr 1978;15:39-44.

7 Webber S, Wilkinson AR, Lindsell D, et al. Neonatal pneumonia. Arch Dis Child 1990;65:207-11.

8 Lehmann D, Heywood P. Effect of birthweight on pneumonia-specific and total mortality among infants in the highlands of Papua New Guinea. P N G Med J 1996;39:274-83.

9 Mathur NB, Garg K, Kumar S. Respiratory distress in neonates with special reference to pneumonia. Indian Pediatr 2002;39:529-37.

10 Mussi-Pinhata MM, Nobre RA, Martinez FE, et al. Early-onset bacterial infection in Brazilian neonates with respiratory distress: a hospital-based study. J Trop Pediatr 2004;50:6-11.

11 Pegues DA, Arathoon EG, Samayoa B, et al. Epidemic gram-negative bacteraemia in a neonatal unit in Guatemala. Am J Infect Control 1994;22:163-71.
12 Rajab A, De Louvois J. Survey of infection in babies at the Khoula Hospital, Oman. Ann Trop Paediatr 1990; 10:39-43.

13 Muhe L, Tilahun M, Lulseged S, et al. Etiology of pneumonia, sepsis and meningitis in infants younger than three months of age in Ethiopia. Pediatr Infect Dis J 1999;18:S56-61.

14 Misra S, Bhakoo ON, Ayyagiri A, et al. Clinical and bacteriological profile of neonatal pneumonia. Indian J Med Res 1991;93:366-70.

15 Singhi S, Singhi PD. Clinical signs in neonatal pneumonia. Lancet 1990;336:1072-3.

16 Isaacs D, Moxon ER. Pneumonia. Handbook of neonatal infections: a practical guide. London: WB Saunders, 2003:151-76.

17 The WHO young infants study group. Bacterial etiology of serious infections in young infants in developing countries: results of a multicentre study. Pediatr Infect Dis J 1999;18:S17-22.

18 English M, Ngama M, Musumba C, et al. Causes and outcome of young infant admisions to a Kenyan district hospital. Arch Dis Child 2003;88:438-43.

19 Gatchalian SR, Quiambao BP, Morelos AM, et al. Bacterial and viral etiology of serious infections in very young Filipino infants. Pediatr Infect Dis $J$ 1999; 18:S50-5.

20 Hoffman JA, Mason EO, Schutze GE, et al. Streptococcus pneumoniae infections in the neonate. Pediatrics 2003;112:1095-102.

21 Duke T, Michael A, Mgone J, et al. Etiology of child mortality in Goroka, Papua New Guinea: a prospective two-year study. Bull World Health Organ 2002;80:16-25.

22 Frank D, Duke T. Congenital syphilis at Goroka Base Hospital: incidence, clinical features, and risk factors for mortality. P N G Med J 2000;43:121-6.

23 Duke T, Blaschke AJ, Sialis S, et al. Hypoxaemia in acute respiratory and nonrespiratory illness in neonates and children in a developing country. Arch Dis Child 2002;86:108-12.

24 Bloland P, Slutsker L, Steketee RW, et al. Rates and risk factors for mortality during the first two years of life in rural Malawi. Am J Trop Med Hyg 1996;55:82-6.

25 Lehmann D, Snnders RC, Marien B, et al. High rates of Chlamydia trachomatis infections in young Papua New Guinean infants. Pediatr Infect Dis $J$ 1999; 18:S62-9.

26 Were FN, Govedi AF, Revathi G, et al. Chlamydia as a cause of late neonatal pneumonia at Kenyatta National Hospital, Nairobi. East Afr Med J 2002;79:476-9.

27 Rosenman MB, Mahon BE, Downs SM, et al. Oral erythromycin prophylaxis vs watchful waiting in caring for newborns exposed to Chlamydia trachomatis. Arch Pediatr Adolesc Med 2003;157:565-71.

28 Preece PM, Anderson JM, Thompson RG. Chlamydia trachomatis infection in infants: a prospective study. Arch Dis Child 1989;64:529.

29 Prevedoros HP, Lee RP, Marriot D. CPAP, effective respiratory support in patients with AIDS-related Pneumocystis carinii pneumonia. Anaesth Intens Care 1991:19:561-6.

30 Weber MW, Mulholland EK, Greenwood BM. Respiratory syncitial virus infection in tropical and developing countries. Trop Med Int Health 1998:3:268-80.

31 Weber MW, Milligan P, Sanneh M, et al. An epidemiological study of RSV infection in the Gambia. Bull World Health Organ 2004;80:562-8.

32 Pillay T, Adhikari M, Mokili J, et al. Severe, rapidly progressive human immunodeficiency virus type 1 disease in newborns with coinfections. Pediatr Infect Dis J 2001;20:404-10.

33 Aiken CG. HIV-1 infection and perinatal mortality in Zimbabwe. Arch Dis Child 2004;67:595-9.

34 Adhikari M, Pillay T, Pillay DG. Tuberculosis in the newborn: an emerging disease. Pediatr Infect Dis J 1997;16:1 108-12.

35 Phadke MA, Gadgil B, Bharacha KE, et al. Replacement-fed infants born to HIV-infected mothers in India have a high early postpartum rate of hospitalization. J Nutr 2003;133:3153-7.

36 Rajesh VT, Singhi S, Kataria S. Tachypnoae is a good predictor of hypoxia in acutely ill infants under 2 months. Arch Dis Child 2000;82:46-9

37 English M, Mohammed S, Ross A, et al. A randomised, controlled trial of once daily and multi-dose gentamicin in young Kenyan infants. Arch Dis Child 2004;89:665-9.

38 Driessen $M$, Ellis JB, Cooper PA, et al. Fluconazole vs. amphotericin B for the treatment of neonatal fungal septicemia: a prospective randomized trial, Pediatr Infect Dis J 1996:15:1107-12.

39 World Health Organization. Management of the child with a serious infection or severe malnutrition: guidelines for care at the first-referral level in developing countries. Geneva: WHO, 2000

40 Duke T, Michael A. Increase in sepsis due to multi-resistant enteric gramnegative bacilli in Papua New Guinea. Lancet 1999;353:2210-11.

41 Musoke RN, Revathi G. Emergence of multidrug-resistant gram-negative organisms in a neonatal unit and the therapeutic implications. J Trop Paediatr 2000;46:86-91.

42 Aurangzeb B, Hameed A. Neonatal sepsis in hospital-born babies: bacterial isolates and antibiotic susceptibility patterns. I Coll Physicians Surg Pak 2003; 13:629-32

43 Rahman S, Hameed A, Roghani MT, et al. Multidrug resistant neonatal sepsis in Peshawar, Pakistan. Arch Dis Child Fetal Neonatal Ed 2000;87:F52-4

44 Sazawal S, Black RE, the Pneumonia Case Management Trials Group. Effect of pneumonia case management on mortality in neonates, infants, and preschool children: a meta-analysis of community-based trials. Lancet Infect Dis 2003;3:547-56.

45 Bang AT, Bang RA, Morankar VP, et al. Pneumonia in neonates: can it be managed in the community? Arch Dis Child 1993;68:550-6.

46 Bang AT, Bang RA, Baitule SB, et al. Effect of home-based neonatal care and management of sepsis on neonatal mortality: field trial in rural India. Lancet 1999;354:1955-61. 
47 Duke T, Willie L, Mgone JM. The effect of introduction of minimal standards of neonatal care on in-hospital mortality. P N G Med J 2000;43:127-36.

48 Duke T, Frank D, Mgone J. Hypoxaemia in children with severe pneumonia in Papua New Guinea. Int J Tuberc Lung Dis 2000:5:51 1-19.

49 Frey B, Shann F. Oxygen administration in infants. Arch Dis Child Fetal Neonatal Ed 2003;88:F84-8.

50 Dhawan A, Narang A, Singhi S. Hyponatremia and the inappropriate ADH syndrome in pneumonia. Ann Trop Paediatr 1992;12:455-62.

51 Duke T, Molyneux EM. Intravenous fluids for seriously ill children: time to reconsider. Lancet 2003;362:1320-3.

52 Kenyon S, Boulvain M, Neilson J. Antibiotics for preterm rupture of membranes. Cochrane Database Syst Rev 2001;4:CD001058.

53 Department of Reproductive Health and Research. Prelabour rupture of membranes. Managing complications in pregnancy and childbirth: a guide for midwives and doctors. World Health Organization WHO/RHR/00.7, 2003:S136-7.

54 Cesar JA, Victora CG, Barros FC, et al. Impact of breast feeding on admission for pneumonia during postneonatal period in Brazil: nested case-control study. BMJ 1999;318:1316-20.

55 Kirkwood BR, Gove S, Rogers S, et al. Potential interventions for the prevention of childhood pneumonia in developing countries: a systematic review. Bull World Health Organ 1995;73:793-8.

56 Petdachai W. Nosocomial pneumonia in a newborn intensive care unit. J Med Assoc Thai 2000;83:392-7.

$57 \mathrm{Ng} \mathrm{SP}$, Gomez JH, Lim SH, et al. Reduction of nosocomial infection in a neonatal intensive care unit (NICU). Singapore Med J 1998;39:319-23.

58 Pawa AK, Ramji S, Prakash K, et al. Neonatal nosocomial infection: profile and risk factors. Indian Pediatr 1997;34:297-302.

59 Webster J, Faoagali JL, Cartwright D. Elimination of methicillin-resistant Staphylococcus aureus from a neonatal intensive care unit after hand washing with triclosan. J Paediatr Child Health 1994;30:59-64.

60 Sharek PJ, Benitz WE, Abel NJ, et al. Effect of an evidence-based hand washing policy on hand washing rates and false-positive coagulase negative staphylococcus blood and cerebrospinal fluid culture rates in a level III NICU. $J$ Perinatol 2002;22:137-43.

61 de Man P, Verhoeven BA, Verbrugh HA, et al. An antibiotic policy to prevent emergence of resistant bacilli. Lancet 2000;355:973-8.

62 Shahid NS, Steinhoff MC, Hoque SS, et al. Serum, breast milk, and infant antibody after maternal immunisation with pneumococcal vaccine. Lancet 1995;346:1252-7.

63 Quiambao BP, Nohynek H, Kayhty H, et al. Maternal immunization with pneumococcal polysaccharide vaccines in the Philippines. Vaccine 2003;21:3451-5.
64 Lehmann D, Pomat WS, Riley ID, et al. Studies of maternal immunization with pneumococcal polysaccharide vaccine in Papua New Guinea. Vaccine 2003;21:3446-50.

65 O'Dempsey TJ, McArdle T, Ceesay SJ, et al. Immunization with a pneumococcal polysaccharide vaccine during pregnancy. Vaccine 1996; 14:963-70.

66 Greenwood B. Maternal immuzation in developing countries. Vaccine 2003;21:3436-41.

67 Munoz FM, Englund JA. A step ahead. Infant protection through maternal immunization. Pediatr Clin North Am 2000;47:449-63.

68 Temmerman M, Gichangi P, Fonck K, et al. Effect of a syphilis control programme on pregnancy outcome in Nairobi, Kenya. Sex Transm Infect 2000;76:117-21.

69 Patel A, Moodley D, Moodley J. An evaluation of on-site testing for syphilis Trop Doct 2001;31:79-82.

70 West B, Walraven G, Morison L, et al. Performance of the rapid plasma reagin and the rapid syphilis screening test in the deiagnosis of syphilis in field conditions in rural Africa. Sex Transm Infect 2002;78:282-5.

71 Opai-Tetteh ET, Hoosen AA, Moodley J. Re-screening for syphilis at the time of delivery in areas of high prevalence. S Afr Med J 1993;83:725-6.

72 Taha TE, Biggar RJ, Broadhead RL, et al. Effect of cleansing the birth canal with antiseptic solution on maternal and newborn mortality in Malawi: clinical trial. BMJ 1997;315:216-19.

73 Humphrey JH, Agoestina T, Wu L, et al. Impact of neonatal vitamin A supplementation on infant morbidity and mortality. J Pediatr 1996;128:489-96.

74 Rahmathullah L, Tielsch JM, Thulasiraj RD, et al. Impact of supplementing newborn infants with vitamin $A$ on early infant mortality: community based randomised trial in southern India. BMJ 2003;327:254-9.

75 Coles CL, Rahmathullah L, Kanungo R, et al. Vitamin A supplementation at birth delays pneumococcal colonization in South Indian infants. J Nutr 2001;131:255-61.

76 Pieper CH, Smith J, Maree D, et al. Is nCPAP of value in extreme preterms with no access to neonatal intensive care? J Trop Pediatr 2003;49:148-52.

77 Narendran V, Donovan EF, Hoath SB, et al. Early bubble CPAP and outcomes in ELBW preterm infants. J' Perinatol 2003;23:195-9.

78 Henderson-Smart DJ, Subramaniam P, Davis PG. Continuous positive airway pressure versus theophylline for apnea in preterm infants. Cochrane Database Syst Rev 2001;4:CD001072.

79 Osborn DA, Henderson-Smart DJ. Kinesthetic stimulation versus theophylline for apnea in preterm infants. Cochrane Database Syst Rev 2000; 2:CD000502

80 Comer AM, Perry CM, Figgit DP. Caffeine citrate: a review of its use in apnea of prematurity. Paediatr Drugs 2001;3:61-79. 